\title{
Energy-efficient cooperative spectrum sensing schemes for cognitive radio networks
}

\author{
Nan Zhao ${ }^{1,5}$, Fei Richard Yu ${ }^{2 *}$, Hongjian Sun ${ }^{3}$ and Arumugam Nallanathan ${ }^{4}$
}

\begin{abstract}
Rapidly rising energy costs and increasingly rigid environmental standards have led to an emerging trend of addressing "energy efficiency" aspect of wireless communication technologies. Cognitive radio can play an important role in improving energy efficiency in wireless networks, because from the green perspective, spectrum is a natural resource which should not be wasted but be shared. In this article, we propose two energy-efficient and time-saving one-bit cooperative spectrum sensing schemes, which have two stages in the spectrum sensing process. If the signal-to-noise ratio is high or no primary user exists, only one stage of coarse spectrum sensing is needed, by which the sensing time and energy are saved. Otherwise, the second stage of fine spectrum sensing will be performed to increase the spectrum sensing accuracy. Furthermore, only one-bit decision is sent by each secondary user to minimize the overhead. The second proposed algorithm fully utilizes the local decisions of the coarse detection, and its energy consumption is further reduced with its sensing performance close to the first one. Plenty of simulation is performed, and the results show that the sensing time and energy consumption are both reduced significantly in the proposed schemes.
\end{abstract}

Keywords: Cognitive radio; Cooperative spectrum sensing; Energy efficiency

\section{Introduction}

Cognitive radio (CR) has attracted significant attention as a promising technology to overcome the spectrum shortage problem caused by the current inflexible spectrum allocation policy $[1,2]$. In CR networks, secondary (unlicensed) users (SUs) should sense the radio environment, and adaptively choose transmission parameters according to sensing outcomes to avoid the interference to primary (licensed) users (PUs) [3]. Hence, it is a fundamental issue in CR networks that SUs should be able to efficiently and effectively detect the presence of PUs $[4,5]$.

The existing spectrum sensing techniques can broadly be divided into four categories: matched filter detection [6], cyclostationary detection [7], entropy-based detection [8-10], and energy detection [11,12]. In matched filter detection and cyclostationary detection, SUs should have some knowledge about the primary signal features. Entropy-based detection can solve the noise uncertainty of the spectrum sensing through information entropy. In

*Correspondence: Richard_Yu@carleton.ca

${ }^{2}$ Computer Engineering, Carleton University, Ottawa, ON K1S 5B6, Canada Full list of author information is available at the end of the article
[8], an entropy-based spectrum sensing scheme is proposed by combining the entropy detection in the time domain and the matched filter. However, the matched filter in the scheme needs some necessary knowledge about the primary signal features, which requires additional overhead and even hardly holds in CR, and thus it is not a blind detector. An entropy-based spectrum sensing scheme in the frequency domain based on the spectrum amplitude is proposed in [9] and proved to be robust to the noise uncertainty; however, its performance can still be improved. In [10], a novel entropy-based spectrum sensing scheme in the frequency domain based on the spectrum power density is proposed, and it is proved that it is also robust to the noise uncertainty with better probability of detection and lower computational complexity. Energy detection has widely been applied, since it does not require a priori knowledge of the primary signals, and has much lower computational complexity than the other three detection schemes. Hence, we focus on energy detection for spectrum sensing throughout this article.

Spectrum sensing is a tough task especially when signalto-noise ratio (SNR) is low. To improve the performance of spectrum sensing, cooperative spectrum sensing (CSS),

\section{Springer}

(c) 2013 Zhao et al: licensee Springer. This is an Open Access article distributed under the terms of the Creative Commons

Attribution License (http://creativecommons.org/licenses/by/2.0), which permits unrestricted use, distribution, and reproduction in any medium, provided the original work is properly cited. 
where individual SUs sense the spectrum and send the information to a fusion center to obtain the final decision, has been studied extensively [10,13-15]. In conventional hard combination CSS [13], only one-bit decision is sent to the fusion center by each $\mathrm{SU}$, and its overhead is minimum; however, its performance can still be improved. Soft combination CSS scheme [14] has the optimal performance through using the accurate sensing results from different SUs; however, its overhead is large, which makes it difficult to be implemented in practical networks.

Two-bit overhead combination CSS scheme is proposed in $[10,15]$, which is a trade-off between hard combination and soft combination CSS. In [16], a three-threshold decision-based CSS (TTD-CSS) scheme is proposed, in which the second local decision bit is sent to the fusion center after the failure of the first cooperation to eliminate the sensing failure. However, the performance of TTD-CSS is not improved much compared with the conventional hard combination CSS. In [17], a two-stage spectrum sensing scheme is proposed for multi-channel sensing. It can decrease the average channel sensing time by allowing the spectrum detector to focus on the channels that are more likely to be vacant. A two-stage sensing scheme using energy detection in the first stage and cyclostationary detection in the second stage is designed in [18], and the second stage detection is performed when the decision metric is greater than the threshold. In [19], a two-stage sensing scheme is proposed to minimize the sensing time. Although the above two-stage spectrum sensing schemes can achieve better performance than the single-stage schemes [17-19], none of them considers CSS. A two-stage two-bit CSS (TSTB-CSS) is given in [10], and its performance is improved over the conventional hard combination CSS; however, it uses two-bit overhead, and its sensing time and energy consumption can still be reduced.

Although some excellent works have been done in CSS, most of them focus on achieving high data rate for SU networks while avoiding the interference to PU networks. Consequently, many of these techniques significantly increase system overhead and energy consumption. However, rapidly rising energy costs and increasingly rigid environmental standards have led to an emerging trend of addressing "energy efficiency" aspect of wireless communication technologies [20]. CR can play an important role in improving energy efficiency in wireless networks, because from the green perspective, spectrum is a natural resource which should not be wasted but be shared. CRs enable this paradigm with smart operation and agile spectrum access. Therefore, the "green" requirements of CR should be well satisfied [21-23].

In this article, we propose two time-saving and energyefficient one-bit CSS (TSEEOB-CSS) schemes, which have two stages in the spectrum sensing process. If the SNR is high or no PU exists, only one stage of coarse spectrum sensing is needed, by which the sensing time and energy are saved. Otherwise, the second stage of fine spectrum sensing will be performed to increase the spectrum sensing accuracy. In addition, only one bit decision is sent to the fusion center to minimize the overhead. Simulation results are presented to show that the sensing time and energy consumption are both reduced significantly in the proposed schemes. The distinct features and contributions of this article are as follows.

- We propose a TSEEOB-CSS algorithm, which can improve the energy efficiency of the system with almost the same detection performance as the conventional CSS algorithms.

- Based on the first TSEEOB-CSS algorithm, another algorithm is proposed. The algorithm makes full use of the local decisions of the first stage coarse detection, and it can further reduce the energy consumption of the first TSEEOB-CSS algorithm with reliable detection performance.

- The parameters setting is important to the two proposed algorithms, and it can affect the performance greatly. Therefore, some rules are given in this article to help to set the parameters suitably.

- The sensing time and energy consumption of the proposed schemes are reduced significantly compared to the conventional CSS scheme, and the time-saving and energy-efficiency performance of the proposed two schemes are analyzed theoretically.

- Extensive simulation results are presented to illustrate the effectiveness of the algorithms in reducing energy consumption and the rules of parameters setting in the proposed algorithms.

The remainder of this article is organized as follows. In Section 2, the system model of the CR network is described, and the energy detection and CSS schemes are introduced. In Section 3, two proposed TSEEOB-CSS algorithms are introduced. In Section 4, parameter setting of the algorithms is discussed, and the performance is analyzed. Simulation results are discussed in Section 5. Finally, we conclude this study and present the future work in Section 6.

\section{System model}

We consider CSS in a centralized CR network consisting of a cognitive base station (fusion center) and a number of SUs. In the network, each SU sends its sensing data to the base station, and the base station combines the sensing data from different SUs and makes the final decision on the presence or absence of the PUs. We assume the sensing data are sent from the SUs to the base station 
free of error throughout this article. In this section, energy detection and CSS are introduced.

\subsection{Energy detection}

The target of spectrum sensing in CR network is to determine whether a licensed band is currently occupied by any PUs or not. This can be formulated into a binary hypotheses testing problem as [24]

$$
x(n)= \begin{cases}\omega(n), & H_{0} \\ h(n) s(n)+\omega(n), & H_{1},\end{cases}
$$

where $n=0,1, \ldots, N$; and $N$ is the number of samples. The PU signal, background noise, and received signal are denoted by $s(n), \omega(n)$, and $x(n)$, respectively. $h(n)$ is the impulse response of the channel between the SU and PU. $H_{0}$ represents the absence of primary signal, while $H_{1}$ represents the presence of primary signal. The noise $\omega(n)$ is assumed to be additive white Gaussian noise with zero mean and unit variance (i.e., $\omega(n) \sim N(0,1)$ ). For ease of analysis, we assume that the channel impulse response $h(n)$ is unchanged during the sensing process, i.e., $h(n)=$ $h$ [25]. Mathematically, the problem can be formulated as a binary hypothesis testing as follows:

$$
T=\frac{1}{N} \sum_{n=1}^{N}|x(n)|^{2} \begin{cases}>\lambda & H_{1} \\ <\lambda & H_{0}\end{cases}
$$

where $T$ is the test statistic, and $\lambda$ is the predetermined threshold.

Let $\sigma_{s}^{2}$ and $\sigma_{\omega}^{2}$ denote the transmitted signal power and noise power, respectively, and assume that $\sigma_{\omega}^{2}=1$. Define $\gamma=\sigma_{s}^{2} / \sigma_{\omega}^{2}$ as the SNR value. The local false alarm and detection probability of the SU can be represented as [26]

$$
\begin{aligned}
P_{f} & =\operatorname{Pr}\left(T>\lambda \mid H_{0}\right) \\
& =Q\left((\lambda-1) \sqrt{\frac{N}{2}}\right), \\
P_{d} & =\operatorname{Pr}\left(T>\lambda \mid H_{1}\right) \\
& =Q\left((\lambda-\gamma-1) \sqrt{\frac{N}{2(\gamma+1)^{2}}}\right),
\end{aligned}
$$

where $Q($.$) is the Q$-function.

\subsection{CSS}

We consider a CR network composed of $K$ SUs and a base station (fusion center), as shown in Figure 1. We assume that each SU performs energy detection independently and then sends the local decision to the base station, which will fuse all available local decision information to infer the absence or presence of the PU.

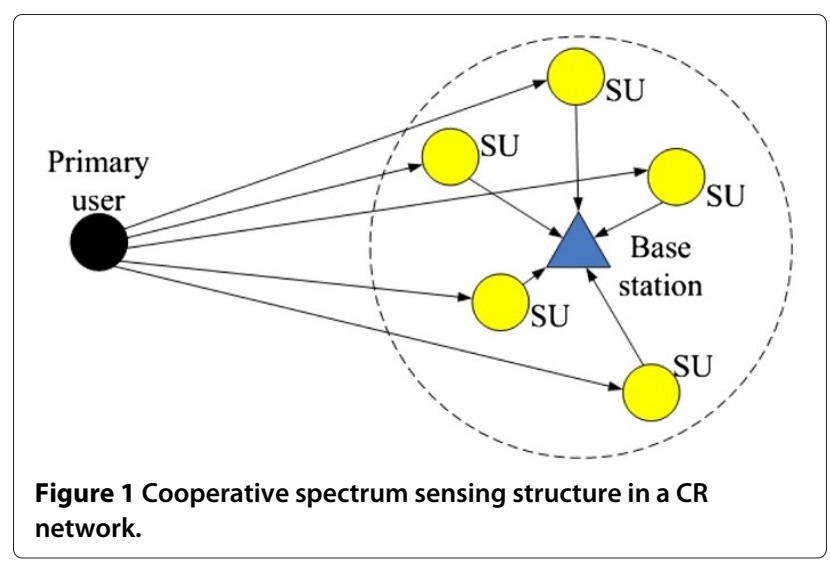

In the conventional hard combination CSS scheme, each cooperative partner $i$ makes a binary decision based on its local observation and then forwards its one-bit decision $D_{i}\left(D_{i}=1\right.$ stands for the presence of the PU, and $D_{i}=0$ stands for the absence of the PU) to the base station. At the base station, all one-bit decisions are fused together according to the logic decision fusion rule $[27,28]$, and the final decision can be obtained as

$$
Y=\sum_{i=1}^{K} D_{i} \begin{cases}\geq k & H_{1} \\ <k & H_{0},\end{cases}
$$

where $H_{0}$ and $H_{1}$ denote the decision made by the base station that the PU is present or absent, respectively. The threshold $k$ is an integer, representing the " $n$-out-of- $K$ " rule. It can be seen that the OR rule corresponds to the case of $k=1$, the AND rule corresponds to the case of $k=K$, and in the VOTING rule $k$ is equal to the minimal integer larger than $K / 2$ [27].

Only one-bit decision information is used in the hard combination CSS, and thus its detection performance is limited. Soft combination CSS scheme uses the accurate sensing results from the SUs, and it can achieve the better performance; however, its overhead is large. Two-bit overhead combination CSS scheme can obtain relatively higher performance than hard combination CSS with lower overhead than soft combination CSS, and it makes a trade-off between hard and soft combination CSS schemes.

\section{TSEEOB-CSS}

The TSTB-CSS algorithm proposed in [10] can improve the performance of the conventional hard combination CSS algorithm; however, its sensing time and energy consumption are the same as those in hard combination CSS. In this article, two TSEEOB-CSS algorithms are presented with almost the same sensing accuracy, and their sensing time and energy consumption are reduced greatly especially when the SNR is high or no PU exists. 
In the proposed algorithms, we try to reduce the energy consumption of conventional CSS scheme with $N_{s}$ samples by designing two TSEEOB-CSS schemes with $\alpha N_{s}$-sample first stage detection and $(1-\alpha) N_{s}$-sample second stage detection. We assume that, in the $N_{s}$-sample detection of these three algorithms, the presence/absence status of the PU does not change. In other words, the received signal is stationary with the observation time $T$ (i.e., $N_{s}$ samples); this assumption is commonly used in the literature [29-32].

\subsection{The first proposed algorithm}

The first proposed two-stage one-bit CSS scheme is shown in Figure 2, and the proposed scheme is represented by the following steps:

Step 1: Perform the first stage coarse energy detection with $\alpha N_{s}$ samples at each SU, where $0<\alpha<0.5$. The sensing result of the $\mathrm{SU}_{i}$ can be calculated as

$$
T 1_{i}=\frac{1}{\alpha N_{s}} \sum_{n=1}^{\alpha N_{s}}\left|x_{i}(n)\right|^{2}
$$

where $x_{i}(n)$ is the $n$th sample of the signal to be sensed at the $\mathrm{SU}_{i}$.

If the detection result of $\mathrm{SU}_{i}(i=1,2, \ldots, K)$

$T 1_{i}>\lambda_{1}+\Delta$, sends the local decision $D 1_{i}=1$ to the fusion center indicating that PUs exist; if $T 1_{i}<\lambda_{1}-\Delta$, sends the local decision $D 1_{i}=0$ to the fusion center indicating that no PU exists; if $\lambda_{1}-\Delta \leq T 1_{i} \leq \lambda_{1}+\Delta$, nothing will be sent. $\lambda_{1}$ and $\Delta$ are two positive parameters that define the upper threshold $\lambda_{1}+\Delta$ and the lower threshold $\lambda_{1}-\Delta$ in the first stage detection.

Step 2: The first stage local decisions $D 1_{i}$ are fused at the fusion center, and the final decision DF can be obtained as

\section{$\mathrm{DF}=\left\{\begin{array}{l}1, \quad \text { More than } K / 2 \mathrm{SUs} \text { indicate presence of PU } \\ 0, \quad \text { More than } K / 2 \mathrm{SU} \text { indicate absence of PU } \\ \text { Final decision cannot be obtained, } \quad \text { Otherwise. }\end{array}\right.$}

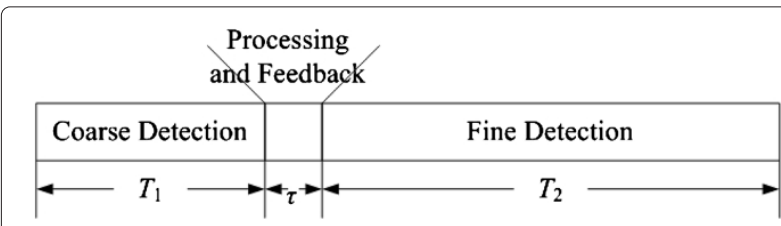

Figure 2 The proposed two-stage one-bit cooperative spectrum sensing scheme.
If the final decision DF can be obtained, DF is sent to each SU. If the final decision DF cannot be obtained, nothing will be done.

Step 3: If the final decision DF is received by the SUs, goes to step 6 . If the final decision DF is not received by the SUs after $\tau$ period, perform the second stage fine energy detection with $(1-\alpha) N_{s}$ samples, and the sensing result of the $\mathrm{SU}_{i}$ can be calculated as

$$
T 2_{i}=\frac{1}{(1-\alpha) N_{s}} \sum_{n=1}^{(1-\alpha) N_{s}}\left|x_{i}(n)\right|^{2}
$$

Assume $\tau \ll T_{1}<T_{2}$ in Figure 2, and thus $\tau$ can be ignored compared with $T_{1}$ and $T_{2}$.

Step 4: Local decision $D 2_{i}(i=1,2, \ldots, K)$ is obtained through the second stage fine energy detection as

$$
D 2_{i}= \begin{cases}1 & T 2_{i} \geq \lambda_{2} \\ 0 & T 2_{i}<\lambda_{2}\end{cases}
$$

where $T 2_{i}$ is the second stage local sensing result of $\mathrm{SU}_{i}$ using energy detection. Then the local decisions $D 2_{i}$ are sent to the fusion center.

Step 5: The second stage local decisions $D 2_{i}$ are fused at the fusion center, and the final decision DF can be obtained according to

$$
\mathrm{DF}= \begin{cases}1 & \sum_{i=1}^{K} D 2_{i} \geq K / 2 \\ 0 & \text { Otherwise. }\end{cases}
$$

DF is sent to each SU, and goes to step 6.

Step 6: Current detection ends.

The first TSEEOB-CSS algorithm described above can achieve almost the same performance as the conventional hard combination CSS algorithm. Its sensing time and energy consumption are reduced obviously when no PU exists or the SNR of PU is high, and therefore the sensing time can be saved and the energy efficiency can be improved effectively.

\subsection{The second proposed algorithm}

The first proposed TSEEOB-CSS algorithm above can achieve better performance with lower energy; however, the sensing results in the first stage coarse detection are not fully utilized, and its energy efficiency can still be improved. Thus, a second TSEEOB-CSS algorithm is proposed based on the first one, and its structure can also be described in Figure 2. The second TSEEOB-CSS scheme is represented by the following steps:

Step 1: Perform the first stage coarse energy detection with $\alpha N_{s}$ samples at each SU as in Equation (6). If the detection result of $\mathrm{SU}_{i}(i=1,2, \ldots, K)$ $T 1_{i}>\lambda_{1}+\Delta$, sends the local decision $D 1_{i}=1$ to 
the fusion center indicating that PUs exist; if $T 1_{i}<\lambda_{1}-\Delta$, sends the local decision $D 1_{i}=0$ to the fusion center indicating that no PU exists; if $\lambda_{1}-\Delta \leq T 1_{i} \leq \lambda_{1}+\Delta$, nothing will be sent.

Step 2: The first stage local decisions $D 1_{i}$ are fused at the fusion center, and the final decision DF can be obtained as in Equation (7).

If the final decision DF can be obtained, DF is sent to each SU. If the final decision DF cannot be obtained, nothing will be done.

Step 3: If the final decision DF is received by the SUs, goes to step 6.

If the final decision DF is not received by the SUs after $\tau$ period, for the SUs that did not obtain the local decision $D 1$ at the first stage, perform the second stage fine energy detection with $(1-\alpha) N_{s}$ samples as in Equation (8), and for the SUs that obtained the local decision at the first stage, no more processing is needed.

Assume $\tau \ll T_{1}<T_{2}$ in Figure 2, and thus $\tau$ can be ignored compared with $T_{1}$ and $T_{2}$.

Step 4: Local decision $D 2_{i}(i=1,2, \ldots, K)$ is obtained through the second stage fine energy detection as

$D 2_{i}= \begin{cases}1 & T 2_{i} \geq \lambda_{2}, \text { when } D 1_{i} \text { was not obtained } \\ 0 & T 2_{i}<\lambda_{2}, \text { when } D 1_{i} \text { was not obtained } \\ D 1_{i}, & \text { when } D 1_{i} \text { was obtained, }\end{cases}$

where $T 2_{i}$ is the second stage local sensing result of $\mathrm{SU}_{i}$ using energy detection, $D 1_{i}$ is the local decision of the $\mathrm{SU}_{i}$ in the first stage. Then the local decisions $D 2_{i}$ are sent to the fusion center.

Step 5: The second stage local decisions $D 2_{i}$ are fused at the fusion center, and the final decision DF can be obtained according to

$$
\mathrm{DF}= \begin{cases}1 & \sum_{i=1}^{K} D 2_{i} \geq K / 2 \\ 0 & \text { Otherwise. }\end{cases}
$$

$\mathrm{DF}$ is sent to each $\mathrm{SU}$, and goes to step 6.

Step 6: Current detection ends.

The first proposed TSEEOB-CSS algorithm can achieve excellent performance with less energy consumption; however, the local decisions of the coarse detection are not fully used. The local decisions of the coarse detection are obtained through two-threshold sensing scheme, and they are more reliable than those of the conventional sensing with the same number of samples. If the local decisions of the coarse detection are utilized in the algorithm, its energy efficiency can be improved. Thus, the second TSEEOB-CSS algorithm is proposed based on the first one, which uses the local decision of the coarse detection to improve the energy efficiency of the algorithm with the same length of sensing time. However, the detection performance of the second TSEEOB-CSS algorithm is worse than the first TSEEOB-CSS algorithm. Thus, we should make a trade-off between the energy efficiency and detection performance to choose the proper algorithm in practical applications.

\section{Threshold setting and performance analysis}

The parameters are important to the proposed algorithms, so in this section, the threshold setting is discussed. In addition, the energy-efficiency and time-saving performance of the algorithms are further analyzed.

\subsection{Analysis of threshold setting}

There are three important parameters in the two proposed TSEEOB-CSS algorithms, $\lambda_{1}, \lambda_{2}$, and $\Delta$. These parameters affect the detection performance greatly. Thus, we discuss the parameters setting, and give some rules as follows.

\section{(1) The rules in setting $\Delta$}

Remark 1.For a fixed value of $\lambda_{1}$, the larger the value of $\Delta$ is, the better the detection performance can be achieved with longer sensing time.

Assume the square of the sampled signal $x(n),|x(n)|^{2}$, follows a distribution with mean $\mu$ and variance $\sigma^{2}$. The sensing result of the first stage detection can be described as

$$
T=\frac{1}{\alpha N_{s}} \sum_{n=1}^{\alpha N_{s}}|x(n)|^{2},
$$

which follows Gaussian distribution with mean $\mu$ and variance $\sigma^{2} /\left(\alpha N_{s}\right)$ according to the central limit theorem. Therefore, the probability the final decision obtained at each SU after the first stage coarse detection can be expressed as

$$
P=1-\int_{\lambda_{1}-\Delta}^{\lambda_{1}+\Delta} \frac{1}{\sqrt{2 \pi \alpha N_{S}} \sigma} \mathrm{e}^{-\frac{(x-\mu)^{2}}{2 \sigma^{2} /\left(\alpha N_{s}\right)}} d x .
$$

Hence, when $\Delta$ is larger, and the probability $(1-P)$ indicating the need of second stage fine detection correspondingly becomes larger, which means the sensing time becomes longer. Also, the detection performance becomes better for the probability of second stage detection is larger.

Remark 2: $\Delta$ should be smaller as the sample number $\alpha N_{s}$ of the first stage coarse detection becomes larger.

Assume the background noise $\omega(n)$ in (1) follows Gaussian distribution with zero mean and unit variance 
(i.e., $\omega(n) \sim N(0,1)$ ), and thus the square of $\omega(n),|\omega(n)|^{2}$, follows chi-square distribution with 1 degree of freedom. The mean of $|\omega(n)|^{2}$ is 1 , and its variance is 2 . The first stage coarse energy detection result can be expressed as

$$
T=\frac{1}{\alpha N_{s}} \sum_{n=1}^{\alpha N_{s}}|\omega(n)|^{2},
$$

when no PU exists, and it follows Gaussian distribution with mean 1 and variance $\sigma_{H 0}^{2}=2 /\left(\alpha N_{s}\right)$ according to the central limit theorem.

We set

$$
\Delta=a \sigma_{H 0}=a \sqrt{2 /\left(\alpha N_{s}\right)}
$$

where $a$ is a positive constant. When the number of samples becomes larger, e.g., $N_{s 2}=2 \alpha N_{s}$, the variance of the first stage coarse energy detection result changes to $2 /\left(2 \alpha N_{s}\right)=1 /\left(\alpha N_{s}\right)$, and hence $\Delta$ should be set as $a \sqrt{1 /\left(\alpha N_{s}\right)}$ accordingly. Therefore, the larger the number of samples is, the smaller the value of $\Delta$ should be.

\section{(2) The rules in setting $\lambda_{1}$}

Remark 3: $\lambda_{1}$ should be set according to the requirement of the false alarm probability $\left(P_{f}\right)$.

In Case I as shown in Figure $3, \lambda_{1}$ is set relatively low (e.g., below 1). If no PU exists, the distribution of the first stage energy detection result is shown in Figure 3 . The probability that the detection result is larger than $\lambda_{1}+\Delta$ is so large that $P_{f}$ cannot be set small (e.g., $P_{f}=0.01$ ). In Case II as shown in Figure $3, \lambda_{1}$ is set relatively high (e.g., above 1). The probability that the detection result is smaller than $\lambda_{1}-\Delta$ is so large that $P_{f}$ cannot be set large (e.g., $P_{f}=0.5$ ). For $P_{f}$ above 0.5 is not meaningful in practical networks, $\lambda_{1}$ is usually set above 1 .

Furthermore, if certain $P_{f}$ can be achieved, the detection performance is better when $\lambda_{1}$ is smaller. However, when $\lambda_{1}$ becomes smaller, the probability indicating the need of second stage fine detection becomes larger when

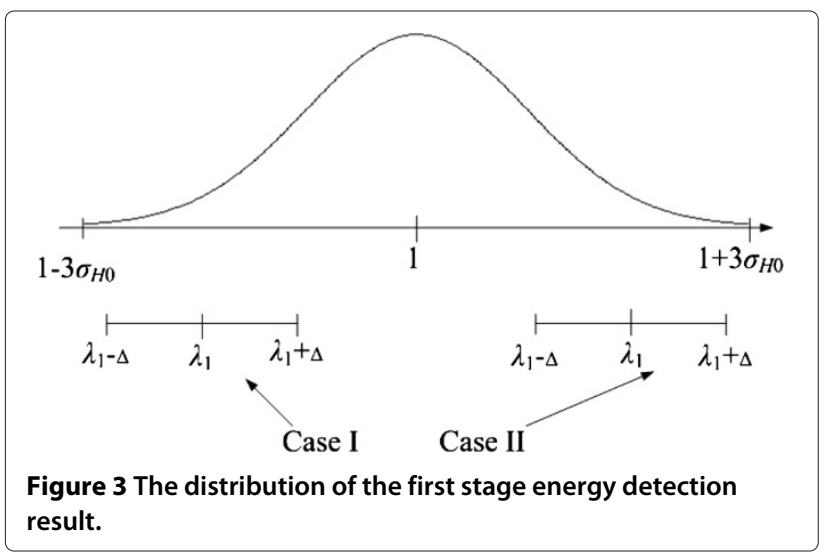

SNR is low or no PU exists, which means the sensing time becomes longer and the energy consumption becomes larger.

\section{(3) The rules in setting $\lambda_{2}$}

As analyzed above, we know that the parameters $\Delta$ and $\lambda_{1}$ determine the detection performance and the computational complexity of the algorithms. Thus, $\Delta$ and $\lambda_{1}$ should be set first according the requirements of the detection performance and energy consumption when applied to practical systems.

$\lambda_{2}$ can be set according to the values of $\Delta$ and $\lambda_{1}$ When $\Delta$ is fixed, $\lambda_{2}$ should be set larger with smaller $\lambda_{1}$. When $\lambda_{1}$ is fixed, $\lambda_{2}$ should be set larger with larger $\Delta$. With certain values of $\Delta$ and $\lambda_{1}$, the value of $\lambda_{2}$ is deterministic. Thus, after $\lambda_{1}$ and $\Delta$ are set, $\lambda_{2}$ can be set according to the required value of $P_{f}$ through measurements in advance.

The above rules in setting $\Delta, \lambda_{1}$, and $\lambda_{2}$ are all suitable for both of the two proposed TSEEOB-CSS algorithms.

\subsection{Analysis of energy-efficiency and time-saving performance}

Remark 4: The energy consumption and sensing time of the proposed schemes are reduced significantly compared to the conventional CSS scheme with the same number of samples, especially when the SNR of the PU signal is high or no PU exists.

The probability of the local decision $D 1_{i}$ in the first stage coarse detection at the $i$ th $\mathrm{SU}$ equal to 1 and 0 can be expressed, respectively, as

$$
\begin{aligned}
P_{1} & =Q\left(\left(\lambda_{1}+\Delta-\gamma-1\right) \sqrt{\frac{\alpha N_{s}}{2(\gamma+1)^{2}}}\right) \operatorname{Pr}\left(H_{1}\right) \\
& +Q\left(\left(\lambda_{1}+\Delta-1\right) \sqrt{\frac{\alpha N_{s}}{2}}\right) \operatorname{Pr}\left(H_{0}\right),
\end{aligned}
$$

$$
\begin{aligned}
P_{0} & =\left(1-Q\left(\left(\lambda_{1}-\Delta-\gamma-1\right) \sqrt{\frac{\alpha N_{s}}{2(\gamma+1)^{2}}}\right)\right) \operatorname{Pr}\left(H_{1}\right) \\
& +\left(1-Q\left(\left(\lambda_{1}-\Delta-1\right) \sqrt{\frac{\alpha N_{s}}{2}}\right)\right) \operatorname{Pr}\left(H_{0}\right),
\end{aligned}
$$

where $\operatorname{Pr}\left(H_{1}\right)$ and $\operatorname{Pr}\left(H_{0}\right)=1-\operatorname{Pr}\left(H_{1}\right)$ denote the probabilities of the presence and absence of the primary signal, respectively.

The energy consumption of the spectrum sensing is mainly caused by the energy detection using the samples of the received signal at all the SUs, and we can define the 
energy consumption of the conventional CSS algorithm through a function of the number of the samples as

$$
E_{\text {CSS }}=K N_{s} \mathrm{e}_{0}
$$

where $K$ is the number of SUs in the CSS, $N_{s}$ is the number of samples at each $\mathrm{SU}$ in the detection, and $\mathrm{e}_{0}$ is the energy consumption corresponding to one sample of the detection.

The energy consumption of the first and second proposed TSEEOB-CSS algorithms can be expressed as

$$
\begin{aligned}
& E_{1}=K \alpha N_{s} \mathrm{e}_{0}+K\left((1-\alpha) N_{s}\right) \mathrm{e}_{0} \\
& \left(1-\sum_{i=\left\lfloor\frac{K}{2}\right\rfloor+1}^{K}\left(\begin{array}{c}
K \\
i
\end{array}\right) P_{1}^{i}\left(1-P_{1}\right)^{K-i}\right. \\
& \left.-\sum_{i=\left\lfloor\frac{K}{2}\right\rfloor+1}^{K}\left(\begin{array}{c}
K \\
i
\end{array}\right) P_{0}^{i}\left(1-P_{0}\right)^{K-i}\right), \\
& E_{2}=K \alpha N_{s} \mathrm{e}_{0}+K\left((1-\alpha) N_{s}\right) \mathrm{e}_{0} \\
& \left(1-\sum_{i=\left\lfloor\frac{K}{2}\right\rfloor+1}^{K}\left(\begin{array}{c}
K \\
i
\end{array}\right) P_{1}^{i}\left(1-P_{1}\right)^{K-i}\right. \\
& \left.-\sum_{i=\left\lfloor\frac{K}{2}\right\rfloor+1}^{K}\left(\begin{array}{c}
K \\
i
\end{array}\right) P_{0}^{i}\left(1-P_{0}\right)^{K-i}\right)\left(1-P_{1}-P_{0}\right) \text {. }
\end{aligned}
$$

From (19)-(21), we can easily obtain

$$
E_{2}<E_{1}<E_{\mathrm{CSS}}
$$

Thus, we can conclude that the energy consumption is reduced significantly by the two proposed CSS schemes, and the energy consumption of the second TSEEOB-CSS algorithm is much lower than that of the first algorithm.

Similarly, assuming the sensing time of the CSS algorithms is mainly determined by the energy detection using the samples of the received signal at all the SUs, the sensing time of conventional CSS algorithm can be defined through a function of the number of the samples as

$$
T_{\mathrm{CSS}}=N_{s} t_{0}
$$

where $t_{0}$ is the duration corresponding to one sample of the detection.
The sensing time of the first and second proposed TSEEOB-CSS algorithms is the same, and can be represented as

$$
\begin{aligned}
& T_{1}=T_{2}=\alpha N_{s} t_{0}+\left((1-\alpha) N_{s}\right) t_{0} \\
&\left(\begin{array}{l}
1 \\
-
\end{array} \sum_{i=\left\lfloor\frac{K}{2}\right\rfloor+1}^{K}\left(\begin{array}{c}
K \\
i
\end{array}\right) P_{1}^{i}\left(1-P_{1}\right)^{K-i}\right. \\
& \\
&\left.-\sum_{i=\left\lfloor\frac{K}{2}\right\rfloor+1}^{K}\left(\begin{array}{c}
K \\
i
\end{array}\right) P_{0}^{i}\left(1-P_{0}\right)^{K-i}\right) .
\end{aligned}
$$

From (23) and (24), we can obtain that

$$
T_{1}=T_{2}<T_{\mathrm{CSS}}
$$

Thus, we can conclude that the time consumption is reduced significantly by the two proposed CSS schemes, and the time consumption of the two algorithms is the same.

The time and energy consumption of the proposed algorithms can be reduced effectively, and we will clarify it briefly. When there are PUs in the network, the first stage coarse energy detection result $T$ follows a distribution with the mean of $1+\mu_{s}$. If the SNR of PU signal is larger, $1+\mu_{s}$ will be larger, and thus the probability that $T>$ $\lambda_{1}+\Delta$ will also become larger. This means the probability indicating the need of second stage fine detection becomes smaller, and thus the sensing time and energy consumption will be reduced due to the low probability of the need of second stage fine detection. When no PU exists in the network, the probability that $T<\lambda_{1}-\Delta$ is relatively large because $\lambda_{1}$ is usually set above 1 . Therefore, the probability indicating the need of second stage fine detection is relatively small, and the sensing time and energy consumption will also be reduced greatly when no PU exists.

From the above analysis, we can conclude that the sensing time and energy consumption is reduced greatly when the SNR of PU signal is high or no PU exists in the proposed two algorithms, and it enables a "green" CR network.

\section{Simulation results and discussions}

In this section, we illustrate the performance of the proposed TSEEOB-CSS algorithms using computer simulations. We assume that the signal of PU is BPSK modulated, and the baseband symbol rate $f_{b}$ is equal to $1 \mathrm{Mbps}$. The sampling frequency $f_{s}$ at SUs is $64 \mathrm{MHz}$. In the TSEEOBCSS algorithms, the number of samples of the two stages, $N_{s}$, is set to $768, \alpha=1 / 3$, and $\Delta$ is set to 0.1 , which is equal to $1.13 \sigma_{H 0}$. 


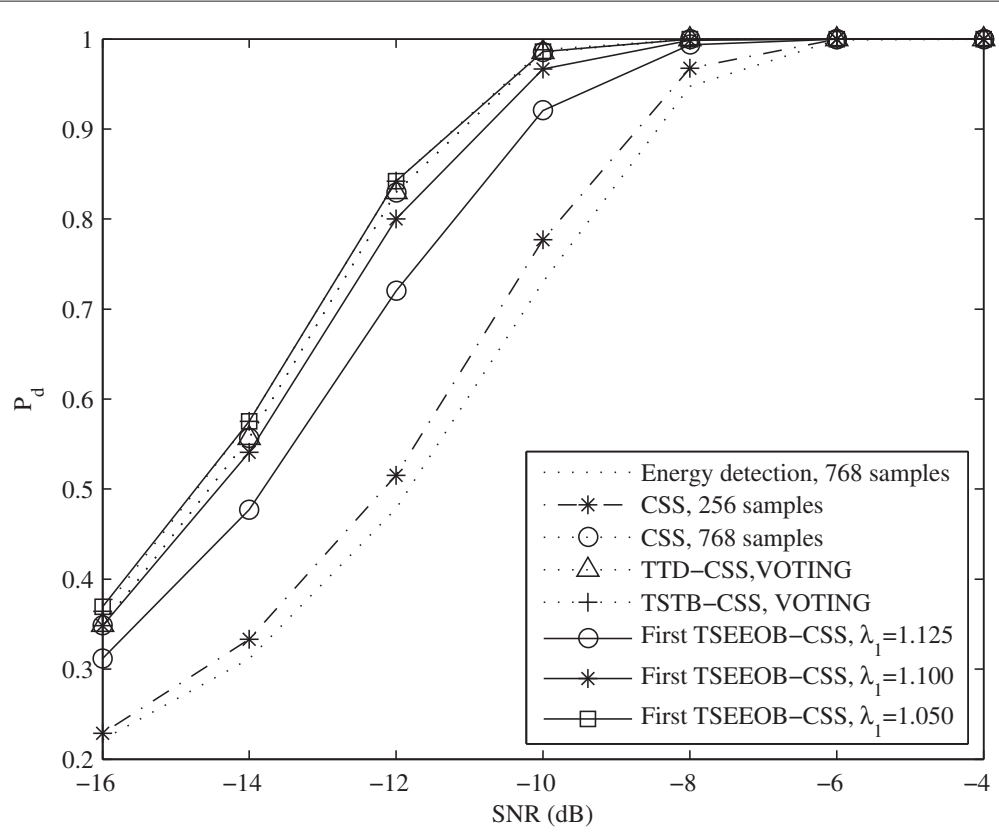

Figure 4 Detection performance against SNR comparison when $P_{f}=0.1$.

First, we compare the detection performance of the first TSEEOB-CSS algorithm with that of TTD-CSS algorithm in [16] and TSTB-CSS algorithm in [10]. In TTD-CSS algorithm, $\lambda$ is set to 1.007 and $\Delta$ is set to 0.1 , the number of samples, $N$, is set to 768 , and VOTING rule is used. In TSTB-CSS algorithm, the number of samples in the first and second stages is both set to $768 / 2=384, \lambda$ is set to 1.034 , and $\Delta$ is set to 0.1 . The detection performance of the energy detection and the hard combination CSS scheme is also compared. The detection probability $\left(P_{d}\right)$ of these algorithms is compared in Figure 4, with unit power of the background noise and $P_{f}=0.1$.

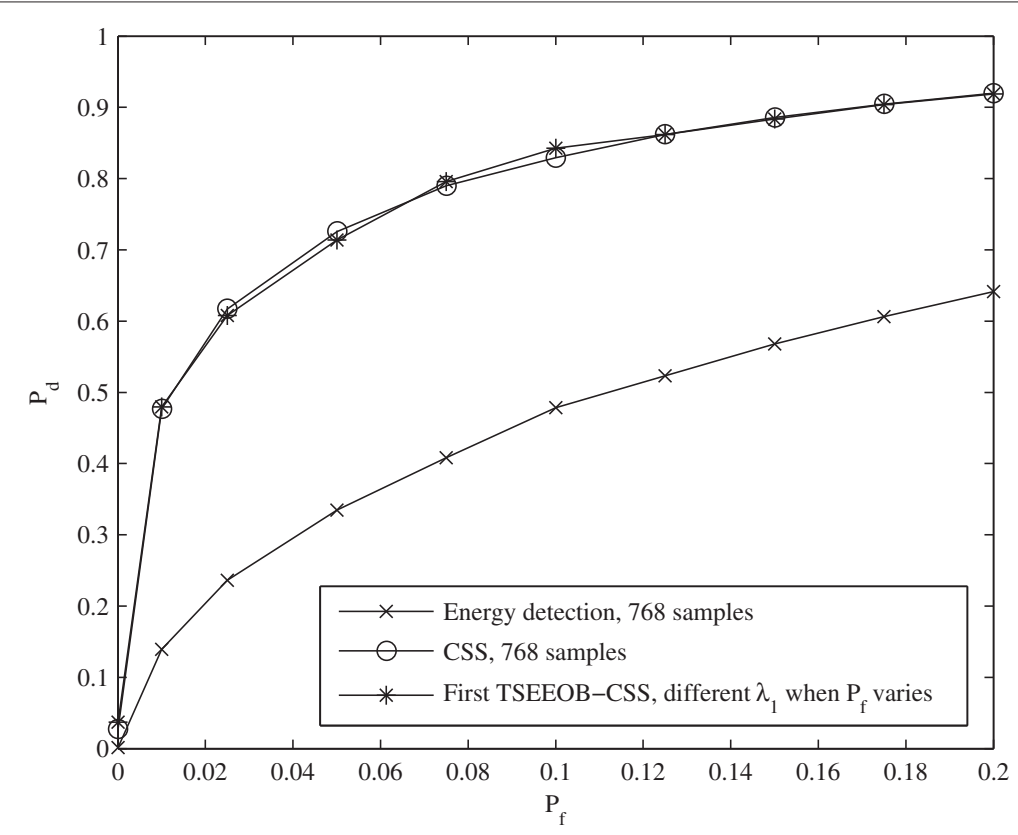

Figure $\mathbf{5}$ ROC curves comparison of the detectors when SNR is equal to $\mathbf{- 1 0} \mathbf{~ d B}$. In the first TSEEOB-CSS algorithm, $\boldsymbol{\lambda}_{1}$ is 1.10 when $P_{f} \in(0,0.05], \lambda_{1}$ is 1.05 when $P_{f} \in(0.05,0.1]$, and $\lambda_{1}$ is 1.00 when $P_{f} \in(0.1,0.2]$. 
Table 1 Parameter $\lambda_{1}$ setting for the first TSEEOB-CSS algorithm with different $\boldsymbol{P}_{\boldsymbol{f}}$

\begin{tabular}{llll}
\hline$P_{f} \in$ & $(0,0.05]$ & $(0.05,0.1]$ & $(0.1,0.2]$ \\
\hline$\lambda_{1}$ & 1.10 & 1.05 & 1.00 \\
\hline
\end{tabular}

From Figure 4 we can see that the performance of the first TSEEOB-CSS algorithm with $\lambda_{1}$ equal to $1.125,1.100$, and 1.050, and corresponding $\lambda_{2}$ equal to 1.024, 1.0295, and 1.033, respectively, is all better than that of 768sample energy detection algorithm and 256-sample hard combination CSS algorithm. The larger the value of $\lambda_{1}$ is, the better performance the first TSEEOB-CSS algorithm can achieve. The detection performance of the first TSEEOB-CSS algorithm with $\lambda_{1}=1.1000$ is close to that of TTD-CSS algorithm, TSTB-CSS algorithm, and 768-sample hard combination CSS algorithm, and its performance is even better than that of these algorithms when $\lambda_{1}$ is equal to 1.050 .

In Section 4, we have analyzed the rules in setting $\lambda_{1}$, and we can conclude that when the required $P_{f}$ is relatively small, $\lambda_{1}$ should be set larger accordingly, and when the required $P_{f}$ is relatively large, $\lambda_{1}$ should be set smaller correspondingly. For example, from the simulation results in Figure 4 we can see that when $P_{f}=0.1$, the detection performance of the first proposed algorithm with $\lambda_{1}=1.05$ is better than that with $\lambda_{1}=1.1$. However, the requirement $P_{f}<0.05$ cannot be achieved when $\lambda_{1}=1.1$, and thus $\lambda_{1}=1.05$ should be selected in this case. Therefore, $\lambda_{1}$ should be set properly according to the required $P_{f}$. The receiver operation characteristic (ROC) curves of the energy detection with 768 samples, the hard combination CSS algorithm with 768 samples and the first TSEEOB-CSS algorithm with 256 samples in the first stage and 512 samples in the second stage are depicted in Figure 5. $P_{f}$ is usually set below 0.2, and thus only part of the ROC curves with $P_{f} \in(0,0.2]$ are given in Figure 5. $\lambda_{1}$ should be set according to the required $P_{f}$, and the $\lambda_{1}$ setting in the simulation of Figure 5 is listed in Table 1.

From the simulations results in Figure 5, we can observe that the ROC performance of the first TSEEOB-CSS algorithm is almost equal to that of the CSS algorithm with different $\lambda_{1}$ set according to the required $P_{f}$ in Table 1 . Thus, the proposed first TSEEOB-CSS algorithm can achieve the same performance as CSS algorithm with much less sensing time and energy consumption.

In Section 3, the second TSEEOB-CSS algorithm is also proposed. It can improve the energy efficiency of the first TSEEOB-CSS algorithm, with its sensing performance a little decrease. The detection performance of the first and second TSEEOB-CSS algorithms is compared in Figure 6 with unit power of the background noise and $P_{f}=0.1$. The detection performance of the CSS algorithm with 768 samples is also adopted in Figure 6.

From the simulation results in Figure 6, we can see that the detection performance of the second TSEEOBCSS algorithm is close to that of the first TSEEOB-CSS algorithm and the CSS algorithm with 768 samples.

A remarkable advantage of the proposed TSEEOB-CSS algorithms is that it can save sensing time and energy

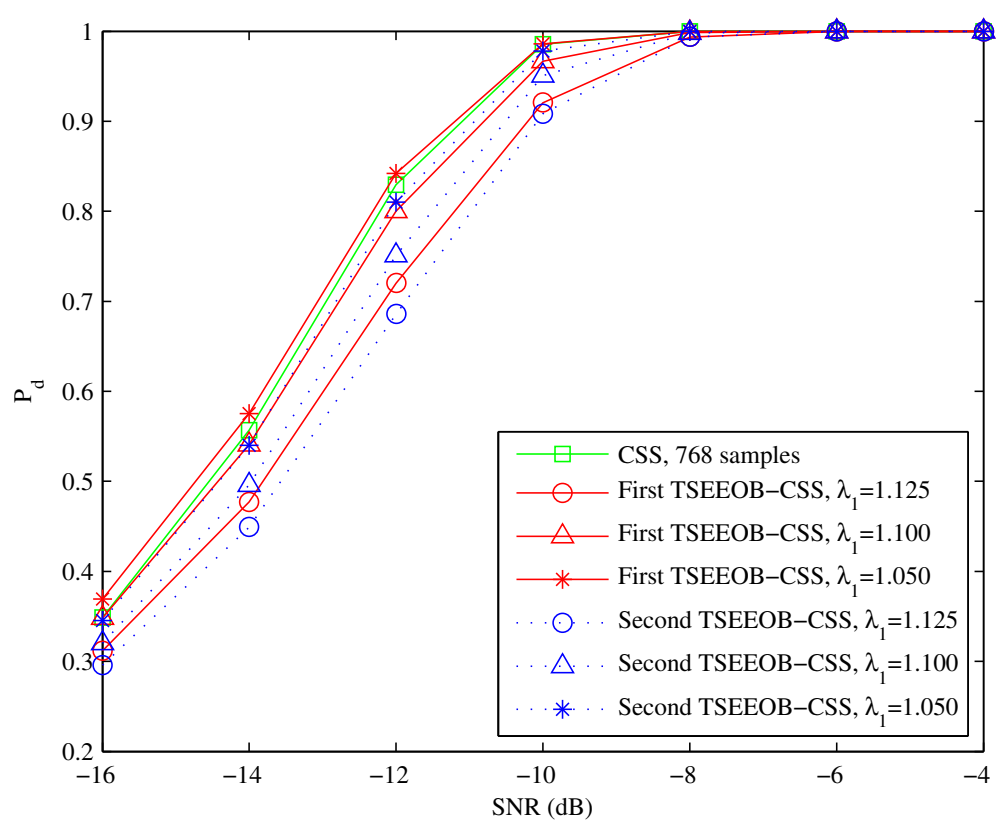

Figure 6 Detection performance comparison between the first and second TSEEOB-CSS algorithms when $P_{f}=0.1$. 




Figure 7 Probability of the second stage detection of the first and second TSEEOB-CCS algorithms with different $\lambda_{1}$ values.

consumption greatly, which will be discussed in the following. The probability of second stage fine detection under different SNRs of the two proposed algorithms is analyzed in Figure 7.

We can see that although the detection performance with $\lambda_{1}=1.125$ is worse than that with $\lambda_{1}=1.100$ and 1.050 (from Figure 4), the probability of second stage detection is the lowest with the shortest sensing time and smallest energy consumption when the SNR is low or no PU exists. Hence, we can conclude that the sensing time is shorter, the energy consumption is smaller, and the detection performance is worse with larger $\lambda_{1}$. In practical CR networks, if the sensing time or energy consumption is the most important factor to be considered, we can use large $\lambda_{1}$; otherwise, $\lambda_{1}$ should be smaller. In addition, the sensing time and energy consumption of the

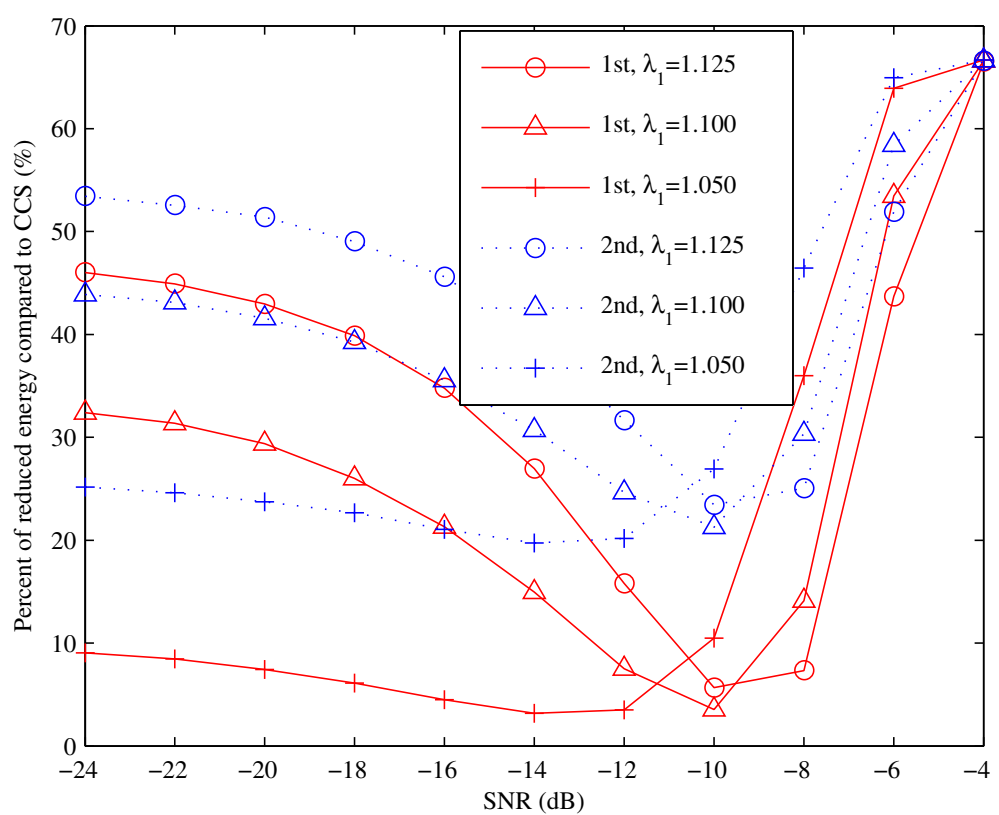

Figure 8 Energy-efficiency performance comparison of the proposed algorithms to the CCS algorithm with different $\lambda_{1}$ values. 


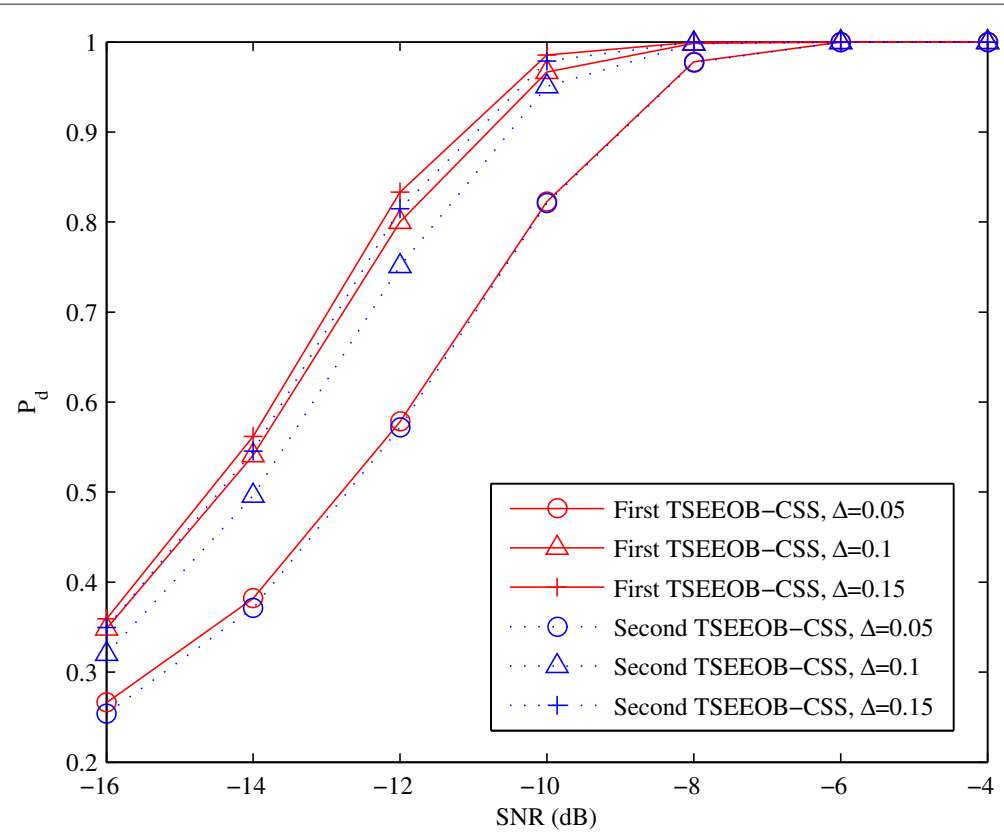

Figure 9 Detection performance comparison of the two proposed algorithm with different values of $\Delta . \lambda_{1}$ is set to 1.1 in the simulation.

second TSEEOB-CCS algorithm is much lower than those of the first TSEEOB-CCS algorithm, and about $20 \%$ of the energy consumption can be saved in the second stage detection.

Using the probability of second stage fine detection obtained from Figure 7, we can calculate the percent of the reduced processing energy by the TSEEOB-CSS algorithms under different SNRs, and the results are shown in Figure 8.

From Figure 8, we can see that when SNR is larger than $-5 \mathrm{~dB}$, the percentage of the reduced energy of these two proposed algorithm is more than 60 , and the maximal percentage of the reduced energy is 66.67 . When no PU exists in the network, the energy consumption can also be

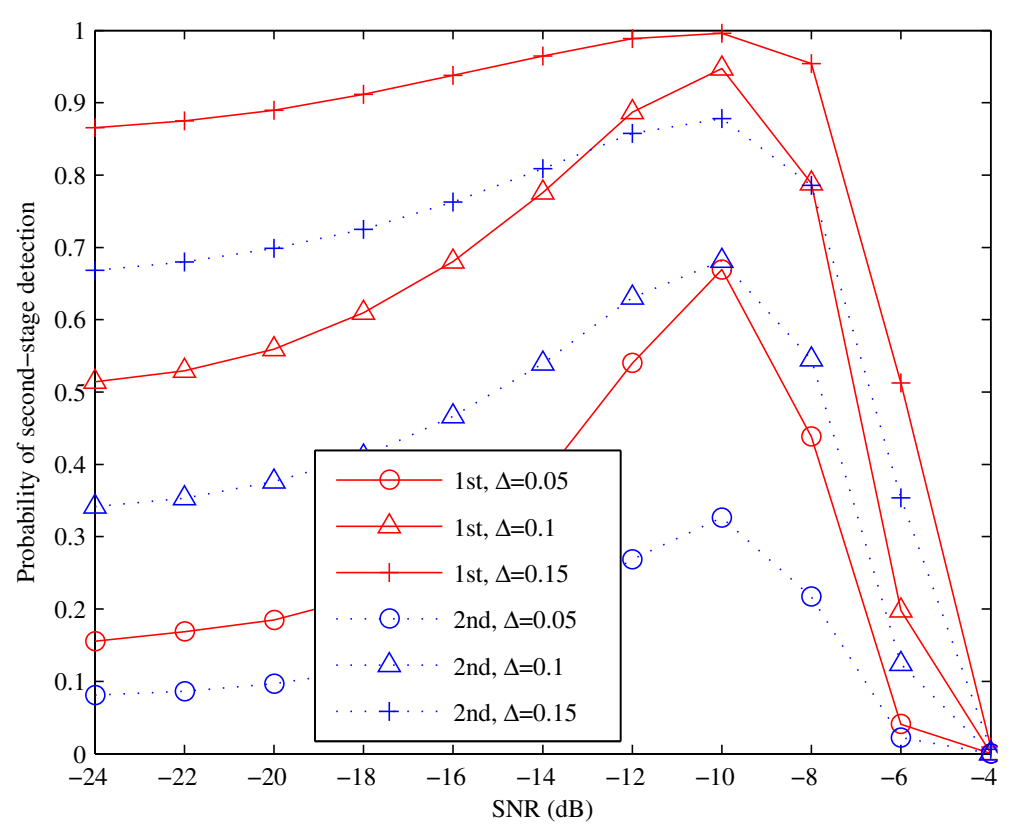

Figure 10 Probability of the second stage detection of the first and second TSEEOB-CCS algorithms with different $\Delta$ values. $\lambda_{1}$ is set to 1.1 in the simulation. 
reduced by 50,35 , and $15 \%$ when $\lambda_{1}$ is set to $1.125,1.100$, and 1.1050, respectively, in the first TSEEOB-CCS algorithm; while in the second TSEEOB-CCS algorithm, the energy consumption can be reduced by 55,45 , and $30 \%$ with these values of $\lambda_{1}$, respectively. Thus, the proposed algorithms can improve energy efficiency of the conventional CSS algorithm significantly with almost the same sensing performance.

In Theorems 1 and 2 of the Section 4, we know that the value of the parameter $\Delta$ affects the detection and energyefficiency performance greatly, so the performance of the algorithms according to $\Delta$ is analyzed. In the following simulation, we set $\lambda=1.1$. The detection performance of the two proposed algorithms with different values of $\Delta$ is shown in Figure 9, and the probability of second stage fine detection with different $\Delta s$ of the algorithms is compared in Figure 10.

The simulation results in Figure 9 have shown that the larger $\Delta$ is, and the better detection performance can be obtained for both the two algorithms. However, it is shown in Figure 10 that when $\Delta$ becomes larger, the probability of second stage fine detection, which also means the energy efficiency becomes lower. Thus, in the practical systems, we should choose appropriate value of $\Delta$ to balance between the detection performance and energy efficiency.

Plenty of simulation results are given in this section, and they have shown the effectiveness of the proposed algorithms. The algorithms can achieve reliable detection performance with much lower energy consumed. The rules in setting the parameters of the two algorithms described in Section 4 are also proved.

\section{Conclusions and future work}

In this article, we have presented two two-stage timesaving and energy-efficient CSS algorithms for CR networks. In the algorithms, only one stage of sensing is needed when the SNR is high or there is no PU. Onebit decision is sent by each SU to minimize the overhead. The second TSEEOB-CSS algorithm fully uses the local decision of the coarse detection, and it can achieve even better energy-efficiency performance with its sensing performance close to the first one and the CSS algorithm. We have also further analyzed the design parameters in the proposed algorithms, and given the rules in setting the parameters. Simulation results were presented to show the effectiveness of the proposed algorithm.

In this article, energy detection is used due to its low computational complexity; however, it cannot avoid the noise uncertainty effectively $[11,33]$. When the background noise fluctuates severely, the performance of the proposed algorithm using energy detection becomes unacceptable. In our previous research work, a spectrum sensing detector based on the entropy of spectrum amplitude with relatively low computational complexity was proposed, and it is robust to the noise uncertainty [10]. The proposed entropy detection algorithm [10] can easily be applied to the proposed TSEEOB-CSS algorithms. In our future work, we will further apply entropybased spectrum detection to the TSOBEE-CSS algorithms proposed in this article, and conduct more research to conquer the problem of noise uncertainty. Moreover, it is interesting to derive closed-form expressions for detection performance and energy efficiency, and analyze the effects of different parameters on the system performance.

\section{Competing interests}

The authors declare that they have no competing interests.

\section{Acknowledgements}

We thank the reviewers for their detailed reviews and constructive comments, which have helped to improve the quality of this article. This study was supported in part by the National Natural Science Foundation of China (NSFC) under Grant no. 61201224, the China Postdoctoral Science Foundation under 2012M510806, and the Natural Science Foundation of Changzhou City under CJ20120015

\section{Author details}

${ }^{1}$ School of Information and Communication Engineering, Dalian University of Technology, Dalian, China. ${ }^{2}$ Computer Engineering, Carleton University, Ottawa, ON K1S 5B6, Canada. ${ }^{3}$ School of Engineering and Computing Sciences, Durham University, Durham, DH1 3LE, UK. ${ }^{4}$ Institute of Telecommunications, King's College London, London, WC2R 2LS, UK. ${ }^{5}$ Changzhou Institute of Dalian University of Technology, Changzhou, China.

Received: 26 September 2012 Accepted: 19 March 2013

Published: 3 May 2013

\section{References}

1. J Mitola, GQ Maguire, Cognitive radio: making software radios more personal. IEEE Pers. Commun. 6(4), 13-18 (1999)

2. Haykin S, Cognitive radio: brain-empowered wireless communications. IEEE J. Sel. Areas Commun. 23(2), 201-220 (2005)

3. Zhao N, Sun H, Robust power control for cognitive radio in spectrum underlay networks. KSII Trans. Internet Inf. Syst. 5(7), 1214-1229 (2011)

4. S Haykin, Thomson D J, JH Reed, Spectrum sensing for cognitive radio. Proc. IEEE. 97(5), 849-877 (2009)

5. T Cui, FF Gao, A Nallanathan, Optimization of cooperative spectrum sensing in cognitive radio. IEEE Trans. Veh. Technol. 60(4), 1578-1589 (2011)

6. HV Poor, An Introduction to Signal Detection and Estimation. (Springer, New York, 1994)

7. N Ghozzi, F Marx, M Dohler, J Palicot, in Cyclostationarilty-based test for detection of vacant frequency bands, vol. 1. Proceedings of the International Conference on Cognitive Radio Oriented Wireless Networks and Communications (Mykonos Island Greece, 2006, 2006), pp. 43-47

8. SV Nagaraj, Entropy-based spectrum sensing in cognitive radio. Signal Process. 89(2), 174-180 (2009)

9. YL Zhang, QY Zhang, T Melodia, A frequency-domain entropy-based detector for robust spectrum sensing in cognitive radio networks. IEEE Commun. Lett. 14(6), 533-535 (2010)

10. N Zhao, A novel two-stage entropy-based robust cooperative spectrum sensing scheme with two-bit decision in cognitive radio. Wirel. Pers. Commun. 69(4), 1551-1565 (2013)

11. D Cabric, SM Mishra, RM Brodersen, in Proceedings of the Asilomar Conference on Signals, Systems, and Computers, vol. 1. Implementation issues in spectrum sensing for cognitive radios, 2004 (Pacific Grove CA, 2004), pp. 772-776

12. YQ Zhao, SY Li, N Zhao, ZL Wu, A novel energy detection algorithm for spectrum sensing in cognitive radio. Inf. Technol. J. 9(8), 1659-1664 (2010) 
13. G Ganesan, $\mathrm{Y} \mathrm{Li}$, in Cooperative spectrum sensing in cognitive radio networks, vol. 1. Proceedings of the International Symposium on New Frontiers in Dynamic Spectrum Acess Networks (Baltimore, MD, 2005, 2005), pp. 137-143

14. J Ma, Y Li, in Proceedings of the IEEE Global Telecommunication Conference, vol. 1. Soft combination and detection for cooperative spectrum sensing in cognitive radio networks (Washington, DC, 2007, 2007), pp. 3139-3143

15. M Mustonen, M Matinmikko, A Mammela, in Cooperative spectrum sensing using quantized soft decision combining, vol. 1. Proceedings of the International Conference on Cognitive Radio Oriented Wireless Networsk in Communication (Hanover, Germany, 2009, 2009), pp. 1-5

16. HX Xia, GP Zhang, HB Xu, A flexible cooperative spectrum sensing scheme for cognitive radio networks. IElCE Electron. Express. 8(8), 542-548 (2011)

17. S Fazeli-Dehkordy, KN Plataniotis, S Pasupathy, in Two-stage spectrum detection in cognitive radio networks, vol. 1. Proceedings of the IEEE International Conference on Acoustics, Speech, and Signal Processing (Dallas, TX, 2010, 2010), pp. 3118-3121

18. S Maleki, A Pandharipande, G Leus, in Two-stage spectrum sensing for cognitive radios, vol. 1. Proceedings of the IEEE International Conference on Acoustics, Speech, Signal and Processing (Dallas, TX, 2010, 2010), pp. 2946-2949

19. PR Nair, AP Vinod, AK Krishna, in A fast two stage detector for spectrum sensing in cognitive radios, vol. 1. Proceedings of the IEEE Vehicular Technology Conference, Fall (San Francisco, CA, 2011, 2011), pp. 1-5

20. GY Li, Z Xu, C Xiong, C Yang, S Zhang, Y Chen, S Xu, Energy-efficient wireless communications: tutorial, survey, and open issues. IEEE Wirel. Commun. 18(6), 28-35 (2011)

21. G Gur, F Alagoz, Green wireless communications via cognitive dimension: an overview. IEEE Netw. 25(2), 50-56 (2011)

22. RXie, FR Yu, H Ji, Y Li, Energy-efficient resource allocation for heterogeneous cognitive radio networks with femtocells. IEEE Trans. Wirel. Commun. 11(11), 3910-3920 (2012)

23. D Grace, J Chen, T Jiang, PD Mitchell, in Using cognitive radio to deliver 'green' communications, vol. 1. Proceedings of the International Conference on Cognitive Radio Oriented Wireless Networks and Communications (Hanover, Germany, 2009, 2009), pp. 1-6

24. FF Digham, MS Alouini, MK Simon, On the energy detection of unknown signals over fading channels. IEEE Trans. Commun. 55(1), 21-24 (2007)

25. SG Z Quan, AH Cui, HV Sayed, Poor, Optimal multiband joint detection for spectrum sensing in cognitive radio networks. IEEE Trans. Signal Process. 57(3), 1128-1140 (2009)

26. H Urkowitz, Energy detection of unknown deterministic signals. Proc IEEE. 55(4), 523-531 (1967)

27. E Visotsky, S Kuffner, R Peterson, in On collaborative detection of TV transmissions in support of dynamic spectrum sharing, vol. 1. Proceedings of the IEEE International Symposium on New Frontiers in Dynamic Spectrum Access Networks (Baltimore, MD, 2005, 2005), pp. 131-136

28. W Zhang, RK Mallik, KB Lataief, in Cooperative spectrum sensing optimization in cognitive radio networks, vol. 1. Proceedings of the IEEE International Conference on Communication (Beijing, China, 2008, 2008), pp. 3411-3415

29. EYC Peh, Y-C Liang, YL Guan, Y Zeng, Optimization of cooperative sensing in cognitive radio networks: a sensing-throughput tradeoff view. IEEE Trans. Veh. Technol. 58(9), 5294-5299 (2009)

30. Z Li, FR Yu, M Huang, A distributed consensus-based cooperative spectrum sensing in cognitive radios. IEEE Trans. Veh. Technol. 59(1), 383-393 (2010)

31. FR Yu, M Huang, H Tang, Biologically inspired consensus-based spectrum sensing in mobile ad hoc networks with cognitive radios. IEEE Netw. 24(3), 26-30 (2010)

32. H Sun, A Nallanathan, N Zhao, C Wang, in Green data transmission in power line communications, vol. 1. Proceedings of the IEEE Global Telecommunication Conference (Anaheim, CA, 2012, 2012), pp. 1-5

33. R Tandra, A Sahai, SNR walls for signal detection. IEEE J. Sel. Topics Signal Process. 2(1), 4-17 (2008)

\section{Submit your manuscript to a SpringerOpen ${ }^{\circ}$ journal and benefit from:}

- Convenient online submission

- Rigorous peer review

- Immediate publication on acceptance

- Open access: articles freely available online

- High visibility within the field

- Retaining the copyright to your article

Submit your next manuscript at $\boldsymbol{\nabla}$ springeropen.com 